\title{
Recent Results on Fusion and Direct Reactions with Weakly Bound Stable Nuclei
}

\author{
A. Shrivastava ${ }^{1, a}$ \\ Nuclear Physics Division, Bhabha Atomic Research Centre, Mumbai 400085, India
}

\begin{abstract}
Recent measurements of fusion and direct reactions in case of weakly bound stable nuclei at extreme sub-barrier energies using a sensitive off beam technique are presented. Deviation in slope of the fusion excitation function, as observed in case of medium heavy systems, is absent in the present asymmetric systems at these low energies. These results along with the study of capture reaction of the breakup fragments using particle- gamma coincidences is presented, thereby giving the current status of the field.
\end{abstract}

\section{Introduction}

Nuclear reactions at energies near the Coulomb barrier display strong inter connectivity among various reaction channels. In heavy ion fusion studies with stable nuclei, the important observations made in the last few decades are the discovery of sub-barrier fusion enhancement and their theoretical description in terms of the couplings to the intrinsic excitations and nucleon transfer of the participating nuclei. Excellent reviews are given by [1]. Recently observed phenomenon of hindrance in fusion reactions in mediumheavy systems at deep sub barrier energies [2] along with availability of low energy radio active ion beams have revived the field of fusion reaction and interest in measurements with weakly bound stable nuclei. The presence of low-lying continuum, short-lived resonance states and low nucleon(s) separation energies in case of weakly bound nuclei, led to the expectation that breakup of the projectile would be a dominant channel to influence the reaction dynamics. Experiments with weakly bound stable nuclei incident on heavy target nuclei have demonstrated that the above-barrier fusion cross-sections are suppressed compared to the expectations for well-bound nuclei [3]. In case of elastic scattering, optical model analysis shows presence of repulsive component in the real part of the potential at near barrier energies [3]. Study of projectile breakup vs transfer followed by breakup [4-6], breakup fusion mechanism [7-9] and phenomenon of fusion hindrance $[2,10]$ are the topics of current interest with weakly bound nuclei.

In this presentation, measurement of fusion cross-section and direct reactions in case of weakly bound stable nuclei using a new sensitive off beam technique will be discussed. First section deals with deep sub-barrier fusion cross-section measurement for ${ }^{6,7} \mathrm{Li}+{ }^{198} \mathrm{Pt}$ followed by the study of fragment capture reaction in case of ${ }^{7} \mathrm{Li}+{ }^{198} \mathrm{Pt}$.

\footnotetext{
a e-mail: aradhana@barc.gov.in
}

\section{Exploring fusion at deep sub barrier with weakly bound nuclei}

Measurements with medium-heavy nuclei highlighted phenomenon of fusion hindrance, observed as a steep change of slope in fusion excitation function and its logarithmic derivative (L(E)) with respect to the coupled channels calculation at deep sub-barrier energies [2]. With the help of a simple and clear picture Dasso and Pollarolo [11] pointed out that the cross-sections at deep sub-barrier energies depend on the shape of the inner part of the inter nuclear potential and could be used as an unique tool to obtain the value of the nuclear potential at small distances (see also [12]). At present there are two successful models that explain a wide range of fusion cross-section data at these low energies. The model proposed by Mişicu and Esbensen is based on the sudden approximation, [13] where a repulsive core is included to take into account the nuclear compressibility arising due to Pauli exclusion principle which modifies the depth and the shape of the minima of the internuclear potential at small distances. Based on an adiabatic picture, a dynamical two-step model via neck formation is proposed by Ichikawa et al. to explain the deep sub-barrier fusion data [14].

Unlike the sharp change in slope of $\mathrm{L}(\mathrm{E})$ as observed in symmetric medium-heavy systems, for asymmetric system a saturation in the slope of $\mathrm{L}(\mathrm{E})$ was observed that was still higher than the values from the coupled channels calculation at the deep sub-barrier [15]. Very recent measurements with medium-light systems showed unsystematic trend in fusion cross-section in nearby systems at these energies $[16,17]$. This unexpected observation together with astrophysical interest shows importance of accurate measurements of fusion cross section at energies deep below the barrier with different entrance channels to understand the phenomenon of fusion hindrance. The fusion of weakly bound nuclei, which is a subject of current interest, has recently been investigated at energies far below the barrier for the weakly bound projectile ${ }^{6} \mathrm{Li}\left(\mathrm{S}_{\alpha / t}\right.$ 
$=1.45 \mathrm{MeV}$ ) on ${ }^{198} \mathrm{Pt}$ [10]. This system incidentally also has a positive Q-value $(8.5 \mathrm{MeV})$. A new sensitive offbeam- $\gamma$-spectroscopy method to obtain the cross-section of residues from fusion, utilizing a coincidence between characteristic KX-rays and $\gamma$-rays from the daughter nuclei, was used [18]. Cross-sections down to a few nano-barns could be measured. Fig. 1 shows fusion cross-sections for ${ }^{6} \mathrm{Li}+{ }^{198} \mathrm{Pt}$. The sum of individual cross-sections for residues formed after $d$-capture $\left({ }^{198-200} \mathrm{Au}\right)$ are also plotted in Fig. 1 . The average angular momenta $(\langle l\rangle)$ derived from the fusion excitation function as suggested in Refs. $[19,20]$ are plotted in Fig. 2(a) along with the logarithmic derivative of the fusion cross-section $(\mathrm{L}(\mathrm{E})=\mathrm{d}[\ln (\sigma \mathrm{E})] / \mathrm{dE})$.

Calculations performed using the coupled-channels (CC) code CCFULL [21] with a standard Woods-Saxon potential (WS) reproduced well both the experimental slope L(E) and the $\langle l\rangle$ values over the entire range of energy. Thus for ${ }^{6} \mathrm{Li}+{ }^{198} \mathrm{Pt}$, the $\mathrm{CC}$ calculations successfully explain the fusion excitation function along with the average angular momentum, consistently implying absence of the fusion hindrance at deep sub-barrier energies. These measurements that extend down to $\mathrm{E}_{c m}=19.8 \mathrm{MeV}$, were well below the threshold energy for observing the fusion hindrance computed from both the approaches (sudden model and adiabatic model) [10].

Single-channel calculations using the above M3Y potential with a repulsive core were also performed as suggested in Ref.[13]. A shallow potential obtained using the M3Y interaction with a repulsive core successfully describe the fusion cross-sections at deep sub-barrier energies for symmetric, asymmetric and positive reaction Q-valued systems $[13,16,22,23]$. But for the present system with a weakly bound projectile this potential does not reproduce the trend of the fusion excitation function, $\mathrm{L}(\mathrm{E})$ and $\langle l\rangle$. It was suggested from the results that the inner part of the interaction potential becomes deeper, going from symmetric to weakly bound asymmetric system, implying reduced contribution of the repulsive core. The actual form of the repulsive core is expected to depend also on the extent of the adiabatic nature of the collision [13].

It is of interest to see whether absence of fusion hindrance in this system arises from the effect of weakly bound cluster structure, transition from the sudden to the adiabatic potential or it is a property of the very asymmetric systems. In order to investigate this, fusion cross-section measurements with ${ }^{7} \mathrm{Li}+{ }^{198} \mathrm{Pt}$ and ${ }^{12} \mathrm{C}+{ }^{198} \mathrm{Pt}$ have been carried out. We present results from measurements of ${ }^{7} \mathrm{Li}+{ }^{198} \mathrm{Pt}$.

Measurement of the excitation function of residues resulting from fusion and direct reactions were performed for ${ }^{7} \mathrm{Li}+{ }^{198} \mathrm{Pt}$ using off-beam $\gamma$-ray counting method. On-line $\gamma$-ray measurement were required to detect residues from $\alpha$-capture. For the offline measurement, beams of ${ }^{7} \mathrm{Li}$ (535 pnA) from Pelletron Linac Facility-Mumbai were bombarded on a ${ }^{198} \mathrm{Pt}$ target in the range of 22 to $45 \mathrm{MeV}$. The targets were self supporting rolled foils of ${ }^{198} \mathrm{Pt}(95.7 \%$ enriched, $\sim 1.3 \mathrm{mg} / \mathrm{cm}^{2}$ thick) followed by an $\mathrm{Al}$ catcher foil of thickness $\sim 1 \mathrm{mg} / \mathrm{cm}^{2}$. Two efficiency calibrated HPGe detectors with a Be window were used for offline $\gamma$ ray counting. The measurements were performed in a low background setup with a graded shielding. The residues in

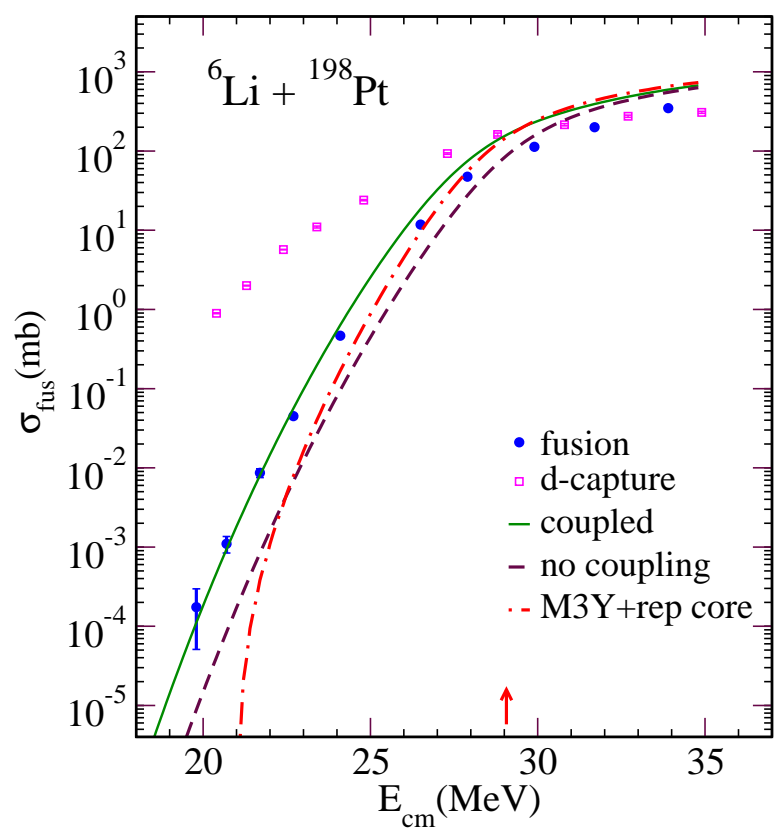

Fig. 1. (color on line) Cross-sections for compound-nuclear fusion and d-capture. The arrow indicates value of the Coulomb barrier $\left(\mathrm{V}_{B}\right)$. The results of the coupled-channels calculations using the WS potential (solid line) along with single channel calculations using the WS potential (dashed line) and the M3Y potential with a repulsive core (dot-dashed lines) are shown (see the text)

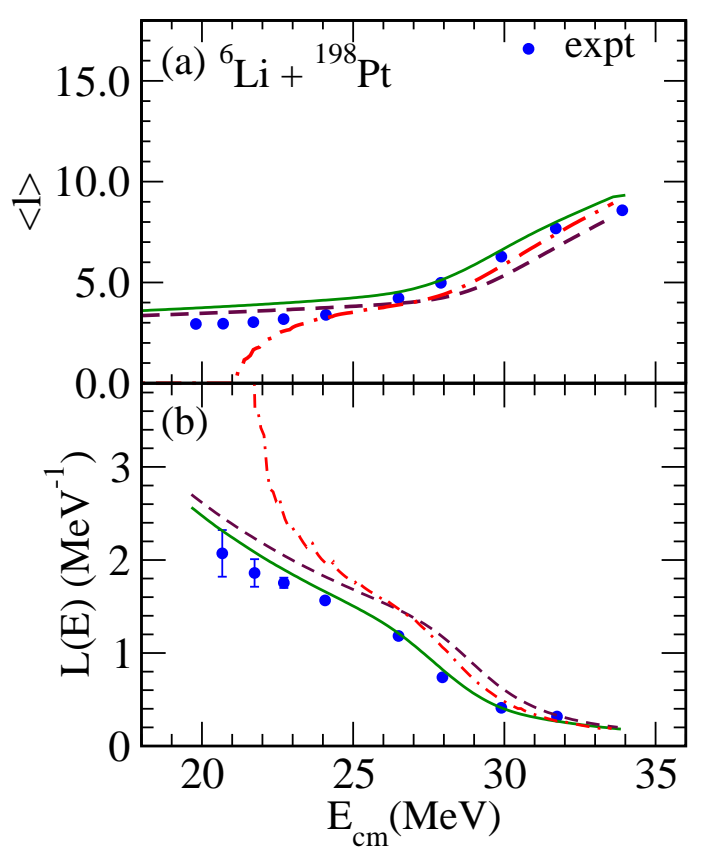

Fig. 2. (color on line) For ${ }^{6} \mathrm{Li}+{ }^{198} \mathrm{Pt}$ (a) Average angular momentum and (b) Logarithmic derivative of the fusion excitation function. The results of the coupled-channels calculations using the WS potential (solid line) along with single channel calculations using the WS potential (dashed line) and the M3Y potential with a repulsive core (dot-dashed lines) are shown in panels (a)(b) (see text) 


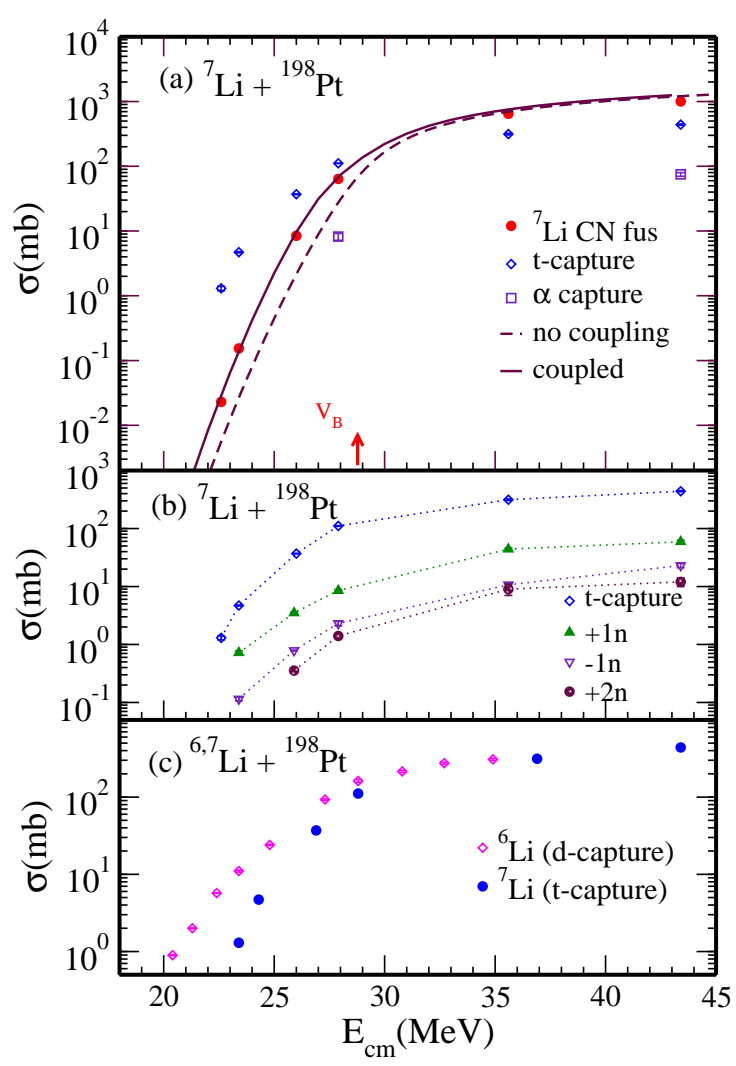

Fig. 3. (color on line) (a) Cross-sections for compound-nuclear fusion and $t$-capture $\left({ }^{198-200} \mathrm{Au}\right)$ for ${ }^{7} \mathrm{Li}+{ }^{198} \mathrm{Pt}$. The arrow indicates value of the Coulomb barrier $\left(\mathrm{V}_{B}\right)$. The results of the coupled-channels calculations (solid line) along with single channel calculations (dashed line) are shown. (b) Comparison of cross-sections from $t$-capture $\left({ }^{198-200} \mathrm{Au}\right), 1$-pickup $\left({ }^{197} \mathrm{Pt}\right)$ and 1n,2n-stripping $\left({ }^{199,200} \mathrm{Pt}\right)$ reactions. (c) Comparison of d-capture and t-capture processes with ${ }^{6} \mathrm{Li}$ and ${ }^{7} \mathrm{Li}$ projectile on ${ }^{198} \mathrm{Pt}$. The dashed lines in panels (b) and (c) are to guide the eye.

the case of fusion $\left({ }^{199-202} \mathrm{Tl}\right)$ were identified by performing $\mathrm{KX}-\gamma$-ray coincidence of the decay radiations from the irradiated sample with detectors placed face to face. Further details of the coincidence method can be found in Ref. [18]. Fusion excitation function for this system is plotted in Fig. 3(a). The $\gamma$-ray yields for residues formed after $t$-capture $\left({ }^{198-200} \mathrm{Au}\right)$ and neutron transfer reactions $\left({ }^{197,199,200} \mathrm{Pt}\right)$ were extracted from inclusive $\gamma$-ray measurements. The total t-capture cross-section (sum of individual residue cross-sections) is shown in Fig. 3 along with 1n, $2 n$ pick up and $1 n$ stripping cross-sections. The error on the data points in Fig. 1 is due to the statistics. The crosssections for the $t$-capture are much larger than those for fusion at deep sub-barrier energies and those for the neutron transfer at all energies. Similar behavior was observed for ${ }^{6} \mathrm{Li}+{ }^{198} \mathrm{Pt}[10]$ and for ${ }^{6} \mathrm{Li}+{ }^{n a t} \mathrm{Pt}$ [24]. The cross-section for $\alpha$-capture forming ${ }^{202 *} \mathrm{Hg}$ could not be measured as the resulting residues are stable (except ${ }^{199 m} \mathrm{Hg}$ ). These crosssections were deduced from the measurement of online prompt gamma transition using four efficiency calibrated and Compton suppressed clover detectors placed at 14.3 $\mathrm{cm}$ from the target position at beam energies of 29 and
$45 \mathrm{MeV}$. The clover detectors were kept at angles of $-55^{\circ}$, $35^{\circ}, 80^{\circ}$, and $155^{\circ}$. Cross-section for the $\alpha$-capture was obtained by adding yields of ${ }^{199} \mathrm{Hg}$ and ${ }^{200} \mathrm{Hg}$ extracted from the $\gamma-\gamma$ coincidence matrix. The coincidence condition reduced the overall background especially due to the same $\gamma$-ray transitions arising from the nuclei that are radioactive and get populated via different channels. The coincidence yield between yrast $\gamma$-ray transitions of the cascade (Eg. 368 and $579 \mathrm{keV}$ in ${ }^{200} \mathrm{Hg}$ ) was obtained by selecting the $2^{+}$to $0^{+}$transition in the clover placed at $55^{\circ}$ and the next $\gamma$-ray transition of the cascade in any of the remaining three clovers. The yield was corrected for the angular correlation between the two E2 transitions and the detector efficiency. The cross-sections so obtained are plotted in Fig. 3(a). The error on the cross-section includes statistics, fitting the angular correlation and efficiency. Cross-section for population of isomeric state of ${ }^{199} \mathrm{Hg}$ obtained from the offline and online method matched well within the errors. The cross-sections for alpha capture are smaller than those for triton capture at both energies ( 29 and $45 \mathrm{MeV}$ ). This can be understood in terms of breakup fusion picture where interaction barrier between $\alpha$ and target is larger than that for triton after breakup of the ${ }^{7} \mathrm{Li}$ projectile. A comparison between fragment-capture cross-sections was made between ${ }^{6} \mathrm{Li}+{ }^{198} \mathrm{Pt}$ and ${ }^{7} \mathrm{Li}+{ }^{198} \mathrm{Pt}$. Larger cross-section of $d$-capture with ${ }^{6} \mathrm{Li}$ compared to $t$-capture with ${ }^{7} \mathrm{Li}$ projectile as shown in Fig. 3(c), could be a consequence of the lower breakup threshold of ${ }^{6} \mathrm{Li}$.

Calculations using the coupled-channels (CC) code CCFULL [21] were performed with the ingoing-wave-boundary condition using a standard Woods-Saxon potential (WS) $\left(\mathrm{V}_{0}=110 \mathrm{MeV}, \mathrm{r}_{0}=1.1 \mathrm{fm}\right.$ and $\left.\mathrm{a}=0.63 \mathrm{fm}\right)$. The calculations included the quadrupole excitation in ${ }^{198} \mathrm{Pt}$, considering coupling in the vibrational model. For ${ }^{7} \mathrm{Li}$ the first excited state was coupled in rotational mode. The results of the calculation with and without the inclusion of the couplings are shown in Fig. 3(a). At energies above the barrier the calculations overestimate the data, as expected from earlier studies involving weakly bound nuclei [25]. As can be seen in the figure, the $\mathrm{CC}$ calculations reproduce the data for energies around and well below the barrier. Fusion hindrance has not been observed in this system in the measured energy range with cross-section at the lowest energy $\approx 2 \mu \mathrm{b}$. The threshold energy $\left(\mathrm{V}_{T}\right)$ for observing fusion hindrance is $\sim 1-2 \mathrm{MeV}$ below the lowest beam energy in the present measurement. Experiment is planned to extend the fusion cross-section data down to energies below this value.

\section{Fragment capture reaction in ${ }^{7} \mathrm{Li}+{ }^{198} \mathrm{Pt}$}

The process of breakup/incomplete fusion where one of the fragments after breakup of the projectile is captured by the target, has been found to be more dominant over one step transfer in case of ${ }^{6} \mathrm{Li}\left({ }^{7} \mathrm{Li}\right)$ for deuteron (triton) capture [7-9]. In case of well bound projectile such processes occur only at high energies $(\approx 10 \mathrm{MeV} / \mathrm{A}$ or more) while for weakly bound nuclei this is the most dominant channel at energies much below the barrier as can be seen in 


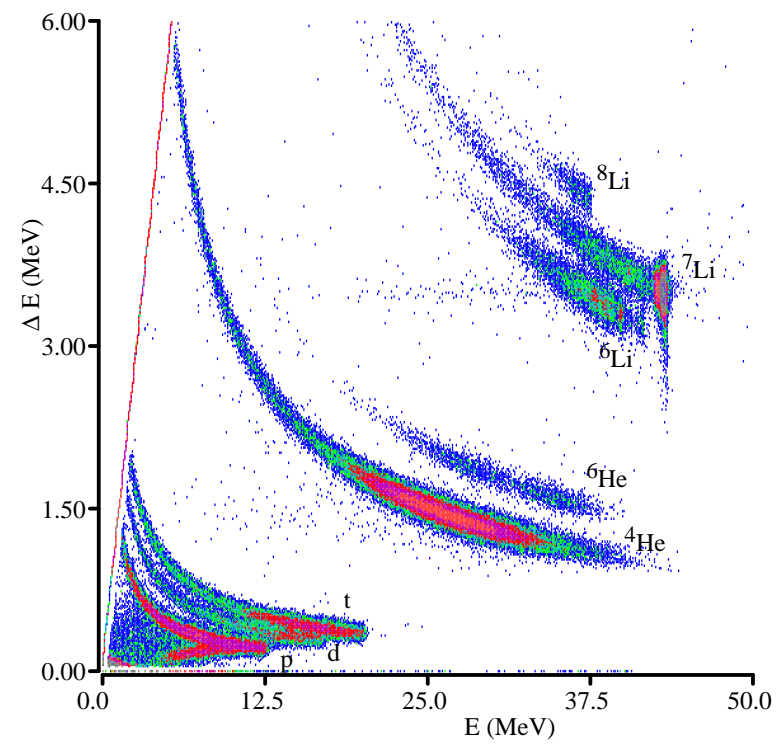

Fig. 4. (color on line) Particle identification plot of energy loss vs total energy obtained in the telescope placed at $\theta_{l a b}=50^{\circ}$ for beam energy of $45 \mathrm{MeV}$. The different reaction products are labeled.

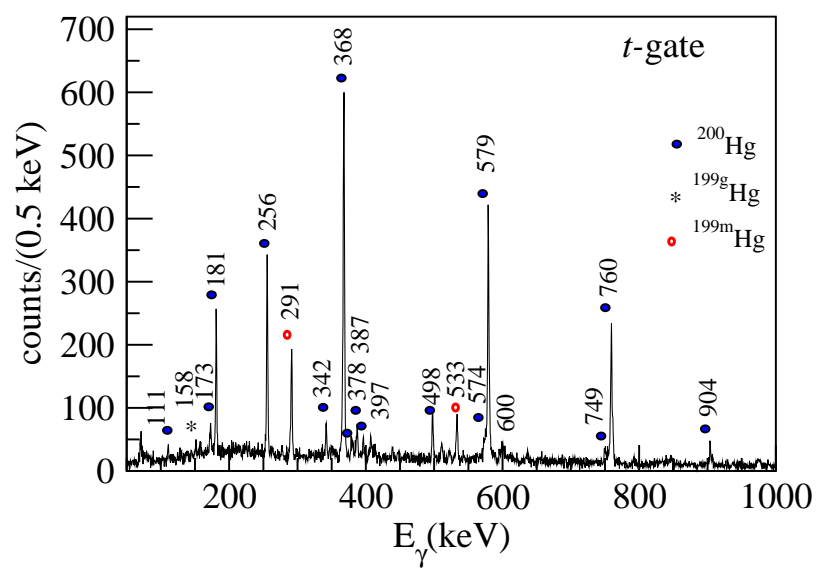

Fig. 5. (color on line) Prompt $\gamma$-ray spectra for $\mathrm{E}_{l a b}=45 \mathrm{MeV}$ obtained in coincidence with outgoing fragments $t$. The $\gamma$-ray transitions originating from the residues from capture of ${ }^{4} \mathrm{He}$ are labeled.

Figs.1,3. The theoretical description of incomplete fusion for weakly bound nuclei has been incorporated in the three dimensional classical trajectory model [26]. This model has been recently modified to give the time evolution of incomplete fusion products and a quantitative description of final products is possible. The present work is aimed to study the capture reaction for the weakly bound clusters, $\alpha$ and $t$, of ${ }^{7} \mathrm{Li}$ using particle-gamma coincidence method.

The experiment was performed at ${ }^{7} \mathrm{Li}$ beam energy of $45 \mathrm{MeV}$, incident on a $1.3 \mathrm{mg} / \mathrm{cm}^{2}$ thick foil of ${ }^{198} \mathrm{Pt}$. Four telescopes $(\Delta \mathrm{E} \sim 25-30 \mu \mathrm{m}$ and $\mathrm{E} \sim 1 \mathrm{~mm})$ at $50^{\circ}, 60^{\circ}, 120^{\circ}$ and $130^{\circ}$ (covering the region near and away from the grazing angle) were used to measure the charged particles produced. Four efficiency calibrated and Compton suppressed clover detectors, to record the coincident $\gamma$-rays, were placed

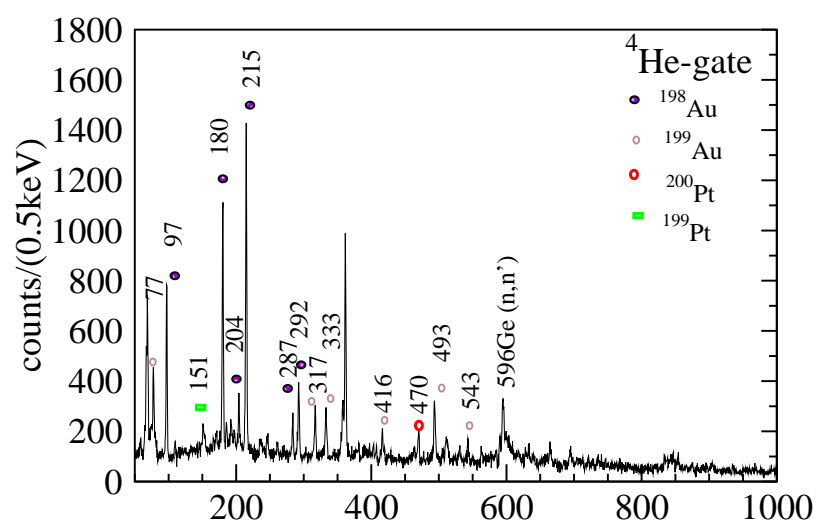

Fig. 6. (color on line) Prompt $\gamma$-ray spectra for $\mathrm{E}_{l a b}=45 \mathrm{MeV}$ obtained in coincidence with outgoing fragment ${ }^{4} \mathrm{He}$. The $\gamma$-ray transitions originating from the residues from capture of $t$ are marked. The $\gamma$-ray arising from $1 \mathrm{n}$ and $2 \mathrm{n}$-transfer $\left({ }^{199,200} \mathrm{Pt}\right)$ are also labeled.

at $14.3 \mathrm{~cm}$ from the target position at angles of $-55^{\circ}, 35^{\circ}$, $80^{\circ}$, and $155^{\circ}$. A fast coincidence between any charged particle detected in the $\Delta \mathrm{E}$ and $\gamma$-rays in any clover detector or a twofold $\gamma$-ray coincidence between clover detectors was used as an event trigger. The individual clovers were operated in add-back mode.

A typical particle identification spectrum for the telescope at forward angle $\left(50^{\circ}\right)$ is shown in Fig. 4. Plotted in Fig. 5,6 is the add-back $\gamma$-ray spectra from the clover detectors generated by selecting outgoing particles detected in telescopes near the grazing angle $\left(50^{\circ}\right.$ and $\left.60^{\circ}\right)$ at the beam energy of $45 \mathrm{MeV}$. The reaction products arising from different channels could be identified by their characteristic $\gamma$-ray transitions. Condition for true coincidence events was obtained using time to amplitude converter spectra between the particle and the $\gamma$-ray. By selecting $t, \gamma$ transitions from residues $\left({ }^{199,200} \mathrm{Hg}\right)$ formed after $\alpha$-capture are seen, and are labeled in Fig. 5. The high-spin states up to $12^{-}$and $14^{+}$respectively for negative and positive parity yrast-levels in ${ }^{200} \mathrm{Hg}$ are observed as reported in Ref. [27] for $\alpha+{ }^{198} \mathrm{Pt}$. The $\gamma$-transitions populating the isomeric state $\left(13 / 2^{+}, \mathrm{T}_{1 / 2} \sim 42.8 \mathrm{~min}\right)$ in ${ }^{199} \mathrm{Hg}$ have relative intensities of $\gamma$-rays similar to the spectra observed with $\alpha$ beam on ${ }^{198} \mathrm{Pt}$ [28]. Gamma spectrum observed with proton gate shows indication of ${ }^{6} \mathrm{He}$ capture.

In Fig. 6, contribution from different reaction channels can be seen with the $\alpha$-gate. The major part of the spectrum is due to residues from $t$-capture $\left({ }^{198,199} \mathrm{Au}\right)$. In case of ${ }^{198} \mathrm{Au}$, the observed $\gamma$-ray transitions from the levels below the isomeric state at $12^{-}$[29] are labeled. Relatively smaller yield of $\gamma$-rays from ${ }^{198,199} \mathrm{Au}$ was observed due to one proton transfer reaction obtained by selecting ${ }^{6} \mathrm{He}$. Both ${ }^{198} \mathrm{Au}$ and ${ }^{199} \mathrm{Au}$ have isomeric states that result in overall loss of the measured yield. The $\gamma$-peaks due to $1 \mathrm{n}$ and $2 \mathrm{n}$-transfer, arising from ${ }^{199} \mathrm{Pt},{ }^{200} \mathrm{Pt}$ are also present in the $\gamma$-ray spectrum with the $\alpha$-gate (Fig. 3(c)). The photo peaks of residues from proton pick-up channel could not be detected due to presence of isomeric states in ${ }^{197}$ Ir. For this nucleus, transitions reported in literature below these states could not be seen and information on tran- 
sitions from states above the isomeric states is not available.

Relative yield of residues formed after alpha and triton capture shows variation for different selected regions of the energy spectra of the outgoing fragment, that corresponds to different excitation energy of the composite system. It will be interesting to compare these observations with the prediction of the classical dynamical model for the incomplete fusion.

\section{Summary}

In this contribution, we have presented the fusion excitation function for very asymmetric systems involving weakly bound projectile at energies well below the barrier. This study shows the absence of fusion hindrance, suggesting modifications in models that explain deep sub barrier fusion data to incorporate weakly bound asymmetric systems. Evaporation residues formed after capture of breakup fragment were identified from coincidence measurements of prompt gamma transitions with the outgoing fragments. Further investigation for the capture of heavy fragment ${ }^{6} \mathrm{He}$ and quantitative understanding of the residues cross-sections from breakup fusion are the next steps in this study.

\section{Acknowledgment}

The author would like to thank all the collaborators for useful discussions while preparing this manuscript and Mumbai Pelltron accelerator staff for providing steady and uninterrupted beam.

\section{References}

1. A.B. Balantekin, N. Takigawa, Rev. Mod. Phys. 70, (1998) 78, M. Dasgupta et al., Ann. Rev. Nucl. Sci. 48, (1998) 401 .

2. C.L. Jiang et al., Phys. Rev. Lett. 89, (2002) 052701.

3. L.F. Canto et al, J. Phys. G 36, (2009) 015109.

4. A. Shrivastava et al. Phys. Letts. B 633, (2006) 463.

5. R. Rafiei et al., Phys. Rev. C 81, (2010) 024601.

6. A. Pakou et al., Phys. Letts. B 633, (2006) 691.

7. V. Tripathi et al., Phys. Rev. C 72, (2005) 0170601.

8. C.M. Castaneda et al., Phys. Letts. B 77, (1978) 371.

9. H. Utsunomiya et al., Phys. Rev. C 28, (1983) 1975.

10. A. Shrivastava et al., Phys. Rev. Letts. 103, (2009) 232702.

11. C. H. Dasso and G. Pollarolo, Phys. Rev. C 68, (2003) 054604.

12. K. Hagino and Y. Watanabe, Phys. Rev. C 76, (2007) 021601(R).

13. S. Mişicu and H. Esbensen, Phys. Rev. Lett. 96, (2006) 112701 ; ibid. Phys. Rev. C 75, (2007) 034606.

14. T. Ichikawa, K. Hagino and A. Iwamoto, Phys. Rev. C 75, (2007) 057603.

15. M. Dasgupta et al., Phys. Rev. Lett. 99, (2007) 192701.
16. C. L. Jiang et al., Phys. Rev. C 82, (2010) (R) 041601.

17. A.M. Stefanini et al., Phys. Rev. C 82, (2010) 017601; A.M. Stefanini, FUSION08, AIP Conf. Proc. 1098, (2009) 3 .

18. A. Lemasson et al., Nucl. Instr. Meth. A 598, (2009) 445 .

19. A.B. Balantekin and P.E. Reimer, Phys. Rev. C 33, (1986) 379.

20. C.V.K. Baba, Nucl. Phys. A 553, (1993) 719c;

V. Tripathi et al., Phys. Rev. Lett. 88, (2002) 172701.

21. K. Hagino et al., Comp. Phys. Comm. 123, (1999) 143.

22. H. Esbensen and S. Mişicu, Phys. Rev. C 76, (2007) 054609.

23. C. L. Jiang et al., Phys. Rev. C 78, (2008) 017601.

24. Yu. E. Penionzhkevich et al., J. Phys. G. Nucl. Part. Phys. 36, (2009) 025104.

25. M. Dasgupta et al., Phys. Rev. C 66, (2002) $041602(\mathrm{R})$.

26. A. Diaz-Torres et al., Phys. Rev. Letts. 98, (2007) 152702.

27. H. Helppi et al., Phys. Rev. C 23, (1981) 2345.

28. D. Mertin et al., Nucl. Phys. A 301, (1978) 365.

29. H.-E. Mahnke, G.Kaindl, F. Bacon and D. Shirley, Nucl. Phys. A 247, (1975) 195. 